\title{
Hemólise interfere na mensuração dos biomarcadores plasmáticos de estresse oxidativo em cães
}

\author{
[Hemolysis interferes in the measurement of plasma biomakers of oxidative stress in dogs] \\ L. Morais, A.M. Bosco, L. Baptisttiolli, R.B.P. Torrecilha, T.C. Valadares, \\ D.J. Hoffmann, P.C. Ciarlini
}

Universidade Estadual Júlio de Mesquita Filho - Araçatuba, São Paulo

\begin{abstract}
RESUMO
Considerando que, entre todas as fontes de erro analítico, a hemólise é a mais importante na rotina laboratorial, o presente estudo teve como objetivo investigar o efeito da hemólise in vitro sobre os principais biomarcadores plasmáticos de estresse oxidativo mensurados (BPEO) de cães. Para tal, amostras de sangue total de 19 cães clinicamente saudáveis foram hemolisadas em diferentes graus por ação mecânica. Amostras controle contendo baixa concentração de hemoglobina $(\mathrm{Hb})$ no plasma foram comparadas com quatro graus de hemólise $(<0,36 ; 0,36-0,60 ; 0,61-1,0 ; 1,1-4 \mathrm{~g} / \mathrm{L} \mathrm{Hb})$. Imediatamente após a hemólise, foram mensuradas as concentrações plasmáticas de ácido úrico (AU), albumina, bilirrubina, gamaglutamiltransferase (GGT), capacidade antioxidante total (TAC) e concentração de oxidante total (TOC). Os erros relativos causados pelos diferentes graus de hemólises foram calculados e confrontados com o erro total aceitável (ETA) e com o limite de erro permitido (LEP) empregados nos programas de controle de qualidade de exames laboratoriais. Foi observado que mesmo pequeno grau de hemólise gera algum erro analítico não aceitável (ETA e/ou LEP) nos BPEO mensurados, exceto na bilirrubina. Foi possível concluir que a hemólise é um fator limitante para avaliação do estresse oxidativo sistêmico mensurado no plasma, podendo causar erros que potencialmente comprometem o diagnóstico clínico.
\end{abstract}

Palavras-chaves: antioxidante, bias, erro analítico, hemoglobina, oxidante

\begin{abstract}
Among all the various sources of analytical error, hemolysis is the most important in the laboratory routine. The present study aimed to investigate the effect of hemolysis "in vitro" on the main plasma biomarkers of oxidative stress (BPEO) dogs. For this purpose, whole blood samples from 19 healthy dogs were hemolyzed in different degrees by mechanical action. Control samples containing low concentration of hemoglobin $(\mathrm{Hb})$ levels in plasma were compared with four degrees of hemolysis $(<0.36$, from 0.36 to 0.60, 0.61 to $1.0,1.1$ to $4 \mathrm{~g} / \mathrm{L} \mathrm{Hb}$ ). Immediately after causing hemolysis, plasma concentrations of uric acid (UA), albumin, bilirubin, gamma glutamyl transferase (GGT), total antioxidant capacity (TAC), and total oxidant concentration (TOC) were measured. The relative errors caused by several levels of hemolysis were calculated and compared with the total acceptable error (TAE) and allowed error limit (LEP) by employees in quality control programs for laboratory tests. Even small levels of hemolysis generate unacceptable analytical error (TAE and / or LEP) in BPEO measured, except for bilirubin. Hemolysis is a limiting factor for the assessment of systemic oxidative stress measured in plasma and may cause errors that potentially compromise clinical diagnosis.
\end{abstract}

Keywords: analytical error, antioxidant, bias, hemoglobin, oxidant

Recebido em 14 de setembro de 2016

Aceito em 7 de agosto de 2017

E-mail: lucianademorais@gmail.com 


\section{INTRODUÇÃO}

O estresse oxidativo é o desequilíbrio entre a produção de oxidantes e as defesas antioxidantes do organismo (Peake e Suzuki, 2004). A produção exagerada e contínua de espécies reativas de oxigênio (ERO) consome as defesas antioxidantes, levando ao dano celular (Zamzami et al., 1996). Os antioxidantes impedem a ação das ERO, de modo a proteger a integralidade e funcionalidade celular (Tavazzi et al., 2000). A mensuração de biomarcadores plasmáticos de estresse oxidativo tem despertado grande interesse científico, em particular nos estudos sobre doenças associadas com excesso de ERO ou diminuição da capacidade antioxidante (Durak et al., 2001).

A hemoglobina é a principal proteína que carreia o oxigênio nos eritrócitos. Para tal, esse tipo celular contém um rico sistema antioxidante capaz de evitar lesões oxidativas e consequente hemólise (Çimen, 2008).

Doenças hemolíticas, coleta e/ou manuseio inadequado das amostras, excesso de pressão na seringa ou no tubo, altas temperaturas, traumas durante a homogeneização ou centrifugação inadequada são as principais causas de hemólise (Lippi et al., 2011).

A liberação de componentes intraeritrocitários devido à hemólise in vivo ou in vitro pode levar a erros analíticos por três diferentes mecanismos: 1. se a concentração eritrocitária do analito for maior que a do plasma, há aumento, se essa condição for inversa, há diminuição; 2. o pigmento da hemoglobina provoca um aumento em sistemas analíticos cujo produto final da reação é mensurado em absorbância com comprimento de onda baixo (300-500nm) e 3. a hemoglobina pode interferir com certas reações químicas, inibindo a diazotização na determinação da bilirrubina ou alterando o pH em medições contínuas de atividade enzimática (Sonntag, 1986).

Muitos laboratórios rejeitam as amostras hemolisadas sem critérios, na maioria das vezes tendo como base uma avaliação visual da amostra. Erros analíticos podem ocorrer em alguns parâmetros bioquímicos de amostras com hemólise moderada ou quase indetectável por inspeção visual (hemoglobina sérica menor que
$0,6 \mathrm{~g} / \mathrm{L})$, de modo que a resposta bastante heterogênea e imprevisível para hemólise em vários parâmetros impede a adoção de medidas corretivas com base no grau de hemólise visual (Lippi et al., 2006). Portanto, há um consenso de que a detecção da hemólise por inspeção visual é demorada, pouco confiável, uma vez que é arbitrária, dificilmente reprodutível, não rastreável e pouco sensível (Simundic et al., 2009). O ponto de corte para considerar uma amostra como sendo hemolisada foi fixado em $0,5 \mathrm{~g} / \mathrm{L}$ de hemoglobina, que é um valor que pode também ser identificado por inspeção visual da amostra (Lippi et al., 2011). A inspeção visual ainda é empregada na maioria dos laboratórios veterinários que não utilizam sistema de automação capaz de quantificar o índice de hemólise (IH). Nos laboratórios humanos, esse sistema ainda está em fase de padronização e validação do IH para se evitarem e se corrigirem os erros (Lippi et al., 2011).

O cálculo do erro causado pela hemólise in vitro obtido mecanicamente tem sido considerado como mais adequado do que outros protocolos hemolíticos (Sonntag, 1986). Para quantificar a interferência da hemólise no erro analítico, vários cálculos têm sido empregados, sendo um dos mais comuns o cálculo de interferência da hemoglobina $(\mathrm{F})$, em que o valor da amostra hemolisada (c) é subtraído do valor da mesma amostra não hemolisada (Co) e dividido pelo valor de $\mathrm{Co}$, de modo que valores maiores que 0,1 são considerados relevantes (Sonntag, 1986). O método analítico e a instrumentação podem ter diferentes sensibilidades ao grau de hemólise para determinados analitos (Yücel e Dalva, 1992). É recomendado que cada laboratório avalie os efeitos da hemólise, uma vez que a interferência varia de acordo com o analisador e o método utilizado (O'Neill e Feldman, 1989).

A prevalência de hemólise em amostras de rotina global é de 3,3\% (Carraro et al., 2000), sendo esta responsável por $40 \%$ a $70 \%$ das amostras inadequadas nos laboratórios humanos (Lippi et al., 2008). Entre todas as várias fontes de erros, a hemólise é a mais importante na rotina laboratorial (Lippi et al., 2011). É provavel que esse viés seja ainda maior nos laboratórios veterinários, diferentemente dos humanos, pois normalmente recebem amostras saguíneas coletadas e transportadas por terceiros. Não obstante, estudos relativos à interferência da 
hemólise sobre o perfil bioquímico plasmático de cães utilizado na rotina clínica veterinária foram amplamente estudados (O’Neill e Feldman, 1989 e Jacobs et al., 1992), porém nenhum desses estudos avaliou o erro relativo causado pela hemólise. São ainda escassas as informações sobre a interferência da hemólise na mensuração dos biomarcadores plasmáticos de estresse oxidativo, em especial na espécie canina (Almeida et al., 2011).

Neste sentido, investigou-se o grau de erro analítico causado pela hemólise in vitro nos principais antioxidantes plasmáticos, na capacidade antioxidante total e na concentração de oxidantes total do plasma de cães.

\section{MATERIAL E MÉTODOS}

O experimento ocorreu de acordo com os princípios éticos em uso de animais do Comitê de Ética em Experimentação Animal da Universidade Estadual Paulista (Protocolo FOA$\mathrm{N}^{\circ}$ 2015/00726).

Numa primeira fase do estudo, foram selecionados 83 cães adultos de diferentes raças e sexo para estabelecer os valores de referência dos marcadores de estresse oxidativo. $\mathrm{Na}$ segunda fase do estudo, outros 19 cães foram selecionados para o ensaio de hemólise mecânica, sendo esse grupo constituído de machos $(n=6)$ e fêmeas $(n=13)$ de diferentes raças e idades (até cinco anos). Todos foram considerados clinicamente saudáveis, sem alterações nos exames físico geral e laboratoriais (hemograma completo e concentração plasmática de albumina, bilirrubina total, ureia, creatinina, colesterol, triglicérides, ácido úrico, aspartato aminotransferase (AST), alanina aminotransferase (ALT), gamaglutamiltransferase (GGT) e fosfatase alcalina). Foram excluídos do estudo animais soropositivos para leishmaniose visceral pelo método ELISA ou tratados nas últimas 72 horas com qualquer medicação.

Após jejum alimentar de oito a 12 horas, $10 \mathrm{~mL}$ de sangue total foram coletados de cada animal por punção da veia jugular, sendo $0,5 \mathrm{~mL}$ acondicionado em tubo com EDTA-K E $_{2}(1 \mathrm{mg}$ $\mathrm{EDTA} / \mathrm{mL}$ sangue) para realização do hemograma completo e $9,5 \mathrm{~mL}$ acondicionados em tubos heparinizados (10UI heparina/mL de sangue), (Vacutainer plus plastic Heparin,
Becton-Dickson, New Jersey, USA) para realização do perfil bioquímico e dos marcadores de estresse oxidativo (ácido úrico, albumina, bilirrubina, GGT, TAC, TOC). Parte do sangue heparinizado $(4,5 \mathrm{~mL})$ foi centrifugado a $1500 \mathrm{G}$ por 10min, e o plasma obtido destinado para a mensuração do perfil bioquímico plasmático.

O hemograma completo foi determinado com auxílio de contador eletrônico de células sanguíneas (BC-2800 Vet, Mindray, China), sendo a contagem diferencial de leucócitos realizada em esfregaços sanguíneos corados com corante hematológico panótico rápido comercial (Instant-Prov, NEWPROV, Pinhais-PR).

Todas as análises bioquímicas plasmáticas foram realizadas em analisador bioquímico automatizado (BS 200, Mindray, China), previamente ajustado com calibrador comercial controle níveis I e II (Biosystems, Barcelona, Spain). Por meio da utilização do conjunto de reativos comerciais (Biosystems, Barcelona, Spain), foram mensurados concentração plasmática de ácido úrico pelo método enzimático (uricase/peroxidase); albumina pelo método do verde de bromocresol; ALT e AST pelo método enzimático UV (glutamato desidrogenase); bilirrubina total pelo método sulfanílico diazotado; colesterol pelo método enzimático (colesterol oxidase/peroxidase); creatinina pelo método cinético (picrato alcalino); proteína total plasmática pelo método do biureto; triglicerídeos pelo método do glicerol fosfato oxidase/peroxidase; e ureia pelo método enzimático UV (urease/glutamato desidrogenase). Além da quantificação da concentração plasmática dos antioxidantes endógenos (albumina, ácido úrico e bilirrubina total), foi determinada a TAC pelo método de inibição de formação de cátion de $\operatorname{ABTS}^{\circledR}$ (2.2'Azino diethyl-bezothiazoline sulfonic acid) descrito por Erel (2004). A TOC no plasma foi mensurada pela reação com íon férrico indicado pelo laranja de xilenol, em meio ácido, conforme descrito por Erel (2005). A concentração da hemoglobina plasmática foi quantificada pelo método ciano-hemiglobina, utilizando-se reagente comercial (Bioclin - Quibasa Quím. Básica Ltda., Belo Horizonte-MG).

Todas as reações bioquímicas foram processadas em duplicata a $37^{\circ} \mathrm{C}$ e dentro de duas horas após a obtenção das amostras. 
Para o ensaio de hemólise mecânica, imediatamente após a coleta, utilizando-se uma pipeta automática, $5 \mathrm{~mL}$ de sangue heparinizado de cada um dos 19 cães foram distribuídos em cinco microtubos de polipropileno tipo Eppendorf, sendo $1 \mathrm{~mL}$ em cada tubo, identificados como H0, H1, H2, H3 e H5. A amostra H0 (controle) não foi submetida ao procedimento de lise mecânica (Tab. 1). Para promover a lise mecânica, com auxílio de uma agulha hipodérmica $(25 \times 0,8 \quad 21 \mathrm{Gx} 1 "$ "SOLIDOR") e seringa de $3 \mathrm{~mL}$, o sangue contido no tubo H1 foi aspirado. Imediatamente após, a agulha foi trocada por uma outra agulha hipodérmica de menor calibre (13x0,45 26G $1 / 2$
"INJEX") e o sangue aspirado foi transferido sob pressão uma única vez para um microtubo de polipropileno. Esse procedimento de aspiração e transferência sob pressão foi repetido duas, três e cinco vezes para se obterem diferentes graus de hemólise nas amostras contidas nos tubos H2, H3 e H5, respectivamente. Em seguida, as amostras de sangue foram centrifugadas a $1500 \mathrm{xg}$ por 10 minutos e o plasma obtido foi protegido da luz.

Para evitar variações temporais, inter e intraensaios, todas as amostras foram processadas laboratorialmente logo após a obtenção do plasma.

Tabela 1. Protocolo de hemólise mecânica. Concentração de hemoglobina livre no plasma obtida de acordo com o número de vezes em que as amostras de sangue foram transferidas sob pressão em seringa acoplada à agulha de pequeno calibre

\begin{tabular}{cccc} 
Grau de hemólise & $\begin{array}{c}\text { Volume de sangue da } \\
\text { amostra }(\mathrm{mL})\end{array}$ & $\begin{array}{c}\text { Quantidade de vezes que o } \\
\text { sangue foi transferido sob } \\
\text { pressão }\end{array}$ & $\begin{array}{c}\text { Concentração final de } \\
\text { hemoglobina contida } \\
\text { no plasma (g/L) }\end{array}$ \\
\hline H0 (controle) & 1.000 & 0 & Não detectável \\
H1 & 1.000 & 1 & $<0.36$ \\
H2 & 1.000 & 2 & $0.36-0.60$ \\
H3 & 1.000 & 3 & $0.61-1.00$ \\
H5 & 1.000 & 5 & $1.01-4.00$ \\
\hline
\end{tabular}

Valores de referência dos biomarcadores de estresse oxidativo plasmáticos de 83 cães saudáveis foram obtidos pelo cálculo do intervalo de confiança 95\%. Para tal, os valores outliers foram excluídos. Para todos os biomarcadores plasmáticos de estresse oxidativo do grupo controle, foi calculado o limite de erro permitido (LEP) estabelecido por Tonks (1963), utilizando-se a seguinte fórmula: $V R>$ $\mathrm{VR}</(\mathrm{VR}<+\mathrm{VR}>) / 2 * 100$, em que $\mathrm{VR}$ corresponde ao valor de referência.

Dos diferentes grupos hemolíticos foi calculado o erro relativo (\%), utilizando-se a seguinte fórmula: $(\mathrm{Vh}-\mathrm{Vn}) / \mathrm{Vn} * 100$, em que $\mathrm{Vh}$ corresponde à amostra hemolisada e $\mathrm{Vn}$ à amostra não hemolisada.

Todas as variáveis foram testadas quanto à normalidade (teste de Kolmogorov-Smirnov) e homocedasticidade (teste Bartlett). O nível de significância das diferenças entre grupos de amostras hemolisadas e o controle foram estimados pelo teste $\mathrm{t}$ pareado, sendo consideradas significativas $\mathrm{p}$ valor $<0,05$ e erro beta $<0,2$. Todas as análises estatísticas foram realizadas com auxílio de um programa computacional estatístico (GraphPad software, version 5.01, 2007), exceto o poder do teste, que foi calculado pelo OpenEpi (versão 3.01, 2013).

\section{RESULTADOS}

Os biomarcadores de estresse oxidativo mensurados no grupo controle corresponderam aos valores de normalidade considerados para a espécie canina (Tab. 2), exceto o ácido úrico e a TAC plasmática, que apresentaram maiores concentrações.

A concentração plasmática de ácido úrico aumentou proporcionalmente ao grau de hemólise, ocorrendo diferenças em baixas concentrações $(<0,36 \mathrm{~g} / \mathrm{L})$ de hemoglobina (Fig. 1A). O erro relativo médio na análise do ácido úrico plasmático foi muito superior aos erros totais aceitáveis (ETA) e ao limite de erro permitido (LEP) de Tonks (1963) para todos os diferentes graus de hemólise (Tab. 2).

A albumina plasmática aumentou significativamente na presença da menor $(<0,36 \mathrm{~g} / \mathrm{L})$ e maior $(1,1-4,0 \mathrm{~g} / \mathrm{L})$ concentração de 
hemoglobina (Fig. 1B), porém nenhum grau de hemólise superou o valor do ETA (Tab. 2). Já concentrações de hemoglobina maiores que $0,36 \mathrm{~g} / \mathrm{L}$ geraram erros superiores aos limites de erro permitido pela ASVCP (Harr et al., 2013) $\left(\mathrm{LEP}_{\mathrm{L}}\right)$ para albumina (Tab. 2). Diferentemente, o erro relativo médio da albumina plasmática em todos os diferentes graus de hemólise ultrapassou o $\mathrm{LEP}_{\mathrm{C}}$ calculado com os valores de referência do próprio laboratório (Tab. 2).

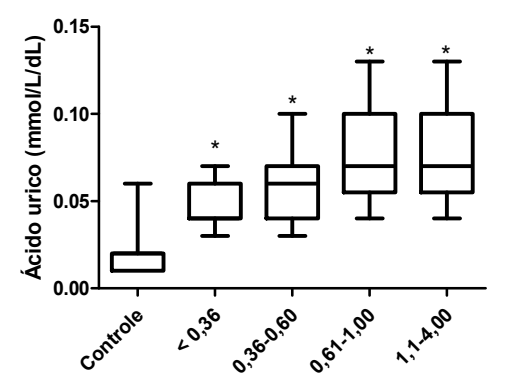

A

Hemoglobina g/L

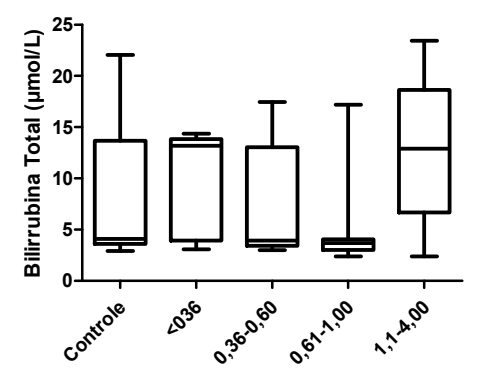

C

Hemoglobina $\mathrm{g} / \mathrm{L}$

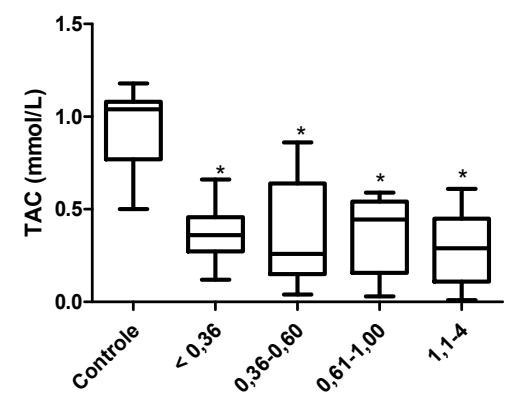

E

Hemoglobina g/L

Figura 1. Boxplot dos marcadores de estresse oxidativo plasmático de cães. Comparação de amostras não hemolisadas (controle) e os diferentes graus de hemólise. Ácido úrico (A), albumina (B), bilirrubina (C), gamaglutamiltransferase (GGT) (D), capacidade antioxidante total (E) e capacidade oxidante total (F). Os valores para o primeiro quartil, a mediana e o terceiro quartil são indicados em cada caixa, e as barras fora da caixa indicam valores mínimos e máximos. *Indica diferença estatística em relação ao controle $(\mathrm{P}<0,05)$.
A hemólise não causou alteração significativa na concentração plasmática de bilirrubina (Fig. 1C), e o erro relativo calculado não superou o ETA e o LEP (Tab. 2).

$\mathrm{Na}$ presença de concentrações de 0,36-1,0g/L de hemoglobina, a atividade de GGT plasmática diminuiu significativamente (Fig. 1D). Em todos os graus de hemólise, o erro relativo médio referente à GGT foi superior ao ETA e ao LEP considerados para a espécie (Tab. 2).

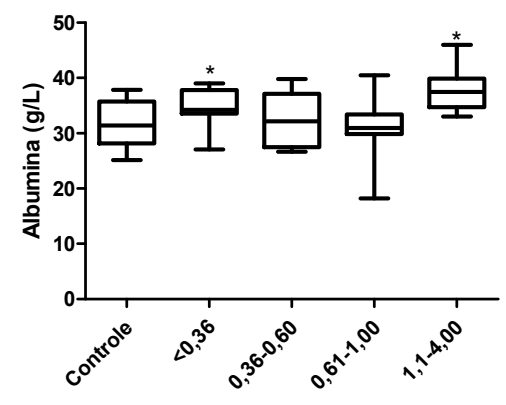

B

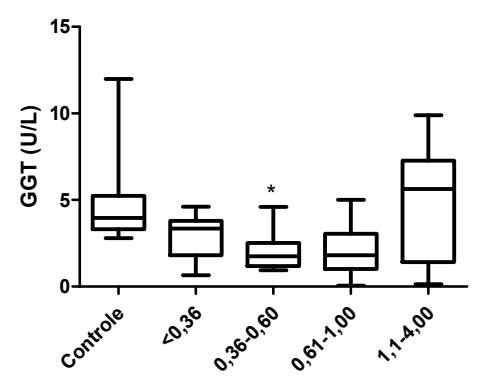

D

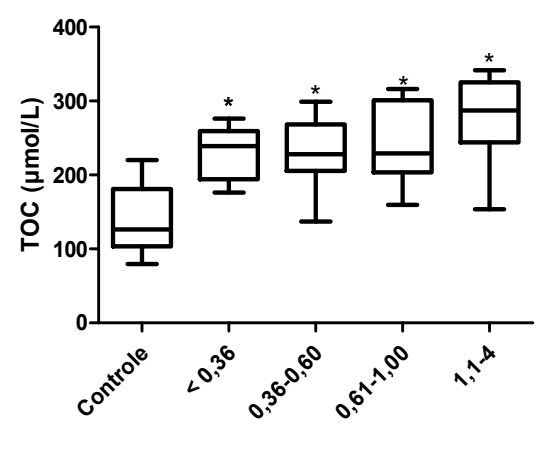

Hemoglobina $\mathrm{g} / \mathrm{L}$ 
Mesmo pequenas concentrações de hemoglobina $(<0,36 \mathrm{~g} / \mathrm{L})$ promovem uma diminuição significativa da TAC plasmática (Fig. 1C) e todos os graus de hemólises causaram erro relativo médio superior ao ETA e ao LEP considerados para a espécie (Tab. 2).
A hemólise não detectável visualmente $(<0,36 \mathrm{~g} / \mathrm{L})$ promoveu um aumento significativo da TOC plasmática (Fig. 1D) e todos os graus de hemólises causaram erro relativo médio superior ao LEP estimado para a espécie (Tab. 2).

Tabela 2. Valores médios e desvios-padrão do grupo controle, valores de normalidade calculados $\left(\mathrm{VN}_{\mathrm{C}}\right)$ e de literatura $\left(\mathrm{VN}_{\mathrm{L}}\right)$, média do erro relativo de acordo com a concentração de hemoglobina livre (Hb), erro total aceitável (ETA), limite de erro permitido de Tonks calculado $\left(\operatorname{LEP}_{\mathrm{C}}\right)$ e de literatura $\left(\mathrm{LEP}_{\mathrm{L}}\right) \mathrm{de}$ diferentes marcadores de estresse oxidativo plasmático de cães

\begin{tabular}{|c|c|c|c|c|c|c|}
\hline & $\begin{array}{c}\text { Ácido } \\
\text { úrico } \\
(\mathrm{mmol} / \mathrm{L})\end{array}$ & $\begin{array}{l}\text { Albumina } \\
(\mathrm{g} / \mathrm{L})\end{array}$ & $\begin{array}{c}\text { Bilirrubina } \\
\text { total } \\
(\mu \mathrm{mol} / \mathrm{L})\end{array}$ & $\begin{array}{l}\text { GGT } \\
\text { (UI) }\end{array}$ & $\begin{array}{c}\mathrm{TAC} \\
(\mathrm{mmol} / \mathrm{L})\end{array}$ & $\begin{array}{c}\text { TOC } \\
(\mu \mathrm{mol} / \mathrm{L})\end{array}$ \\
\hline Controle & $0,38 \pm 0,23$ & $32,08 \pm 3,65$ & $0,29 \pm 0,22$ & $5,27 \pm 2,23$ & $0,69 \pm 0,3$ & $113,65 \pm 55,61$ \\
\hline $\mathrm{VN}_{C}$ & $0,32-0,43$ & $31,22-32,93$ & $0,22-0,35$ & $4,93-5,99$ & $0,59-0,77$ & $97,36-129,93$ \\
\hline $\mathrm{VN}_{\mathrm{L}}$ & $0-0,12^{* 1}$ & $26-33^{*_{1}}$ & $1,71-8,55^{* 1}$ & $1,2-6,4^{* 1}$ & $0,9^{* 2}$ & $200^{* 2}$ \\
\hline Erro relativo & & & & & & \\
\hline $\mathrm{Hb}<0,36 \mathrm{~g} / \mathrm{L}$ & $287,25 \%$ & $4,41 \%$ & $2,04 \%$ & $-34,70 \%$ & $-51,08 \%$ & $79,73 \%$ \\
\hline $\mathrm{Hb} 0,36-0,60 \mathrm{~g} / \mathrm{L}$ & $281,69 \%$ & $7,61 \%$ & $3,57 \%$ & $-42,83 \%$ & $-63,23 \%$ & $80,28 \%$ \\
\hline $\mathrm{Hb} 0,61-1,0 \mathrm{~g} / \mathrm{L}$ & $248,94 \%$ & $6,22 \%$ & $-2,41 \%$ & $-47,60 \%$ & $-65,06 \%$ & $69,36 \%$ \\
\hline $\mathrm{Hb} 1,1-4,0 \mathrm{~g} / \mathrm{L}$ & $636,76 \%$ & $9,92 \%$ & $-0,55 \%$ & $-20,32 \%$ & $-64,73 \%$ & $130,30 \%$ \\
\hline $\mathrm{ETA}^{* 3}$ & $10 \%$ & $15 \%$ & $30 \%$ & $20 \%$ & NR & NR \\
\hline $\mathrm{LEP}_{\mathrm{C}}$ & $4,95 \%$ & $0,89 \%$ & $8,3 \%$ & $3,32 \%$ & $4,52 \%$ & $5,01 \%$ \\
\hline $\mathrm{LEP}_{\mathrm{L}}$ & $50 \%$ & $5,9 \%$ & $33,33 \%$ & $34,21 \%$ & $26,97 \%$ & $19,76 \%$ \\
\hline
\end{tabular}

$\mathrm{VN}_{\mathrm{c}}$ : intervalo de confiança (95\%) do grupo controle; $\mathrm{VN}_{\mathrm{L}}{ }^{* 1}$ Kaneko et al. (2008) e ${ }^{* 2}$ Almeida et al. (2013); ${ }^{* 3}$ ETA Harr et al. (2013); *NR: não relatados.

\section{DISCUSSÃO}

Para um adequado controle de qualidade de um sistema analítico, é essencial ter valores de referência específicos para cada espécie (Jacobs et al., 1992), preferencialmente obtidos com a mesma metodologia, reagentes e equipamentos (Oliveira e Mendes, 2010). Embora existam valores de referência para o perfil bioquímico de cães (Kaneko et al., 2008), estes foram determinados mediante o uso de um conjunto de reagentes e equipamentos diferentes do empregado no presente estudo. Valores plasmáticos de TAC e TOC utilizando a mesma metodologia e equipamento são restritos a estudos experimentais com pequeno tamanho amostral (Almeida et al., 2013). Os valores de normalidade dos biomarcadores de estresse oxidativo estabelecidos no presente estudo foram similares e apresentaram menor amplitude que os descritos na literatura (Almeida et al., 2013; Bosco et al., 2016), exceto o ácido úrico. Essa diferença quanto ao valor médio de ácido úrico e o intervalo de confiança de todos os biomarcadores de estresse oxidativo reforçam a orientação de que cada laboratório deve estabelecer seus próprios valores de referência para adequada interpretação e controle de qualidade.

Valores de referência também são essenciais para o cálculo dos erros analíticos, em particular para estabelecerem o limite de erro permitido (LEP), que é considerado um importante parâmetro para distinguir se um valor é normal ou anormal. $\mathrm{O}$ LEP preconizado por Tonks (1963) estabelece que o limite de variabilidade não pode ultrapassar $25 \%$ do intervalo de confiança do método analítico. A Clinical Laboratory Improvement Amendments (Clinical..., 1988), de modo similar, recomenda que o erro sistemático e o erro aleatório não ultrapassem $50 \%$ e $25 \%$ do erro total aceitável (ETA) baseado em requisitos clínicos (Mendes e Romano, 2010).

Os exames laboratoriais são responsáveis por $65 \%$ a $75 \%$ das informações pertinentes à decisão médica humana (Westgard e Darcy, 2004), sendo a hemólise a principal fonte de erro analítico (Lippi et al., 2008). Não obstante, estudos relativos à interferência da hemólise sobre marcadores de estresse oxidativo na espécie canina são escassos e nenhum levou em consideração o LEP. Para melhor interpretação 
do erro relativo causado pela hemólise em cães, o presente estudo confrontou o LEP calculado no laboratório com o LEP calculado com valores de literatura e o ETA estabelecido pela ASVCP Guidelines Allowable Total Error (Harr, 2013).

O aumento do ácido úrico devido à hemólise foi anteriormente descrito em humanos (Yucel e Dalva, 1992), equinos e bovinos (Almeida et al., 2011), porém há relato de que a hemoglobina humana causa decomposição prematura do peróxido de hidrogênio necessário para a reação que quantifica o ácido úrico (Yucel e Dalva, 1992). No presente estudo, observou-se que, em cães, a concentração plasmática de ácido úrico aumenta proporcionalmente ao grau de hemólise, gerando um erro relativo médio muito superior ao ETA admitido pela ASVCP Guidelines Allowable Total Error (Harr, 2013) e ao LEP. Esses resultados são concordantes com a maioria dos estudos realizados em outras espécies e acrescentam que tal interferência compromete a interpretação clínica dos resultados de ácido úrico plasmático canino.

Acredita-se que o erro analítico na mensuração da albumina sérica canina varie não só com o grau de hemólise como também com a metodologia e o equipamento utilizado na mensuração (O'neill e Feldman, 1989). O aumento dos valores de albumina em plasma hemolisado observado neste estudo já havia sido relatado em amostras séricas de cães (Leard et al., 1990; Almeida et al., 2011) e humanos (Koseoglu et al., 2011). No presente estudo, observou-se que nenhum grau de hemólise superou o ETA aceito pela ASVCP (Harr, 2013) para albumina, porém o erro relativo médio da albumina plasmática em todos os diferentes graus de hemólise ultrapassou o $\mathrm{LEP}_{\mathrm{C}}$ calculado com os valores de referência do próprio laboratório, e o mesmo fato ocorreu em relação ao $\mathrm{LEP}_{\mathrm{L}}$ estimado da literatura quando as concentrações foram maiores ou iguais a 0,36 $\mathrm{g} / \mathrm{L}$ de hemoglobina. Esses resultados confirmam que a decisão de rejeição de uma amostra hemolisada pode variar com o critério adotado (ETA ou LEP). Fica evidente que o LEP estabelecido pelo próprio laboratório para a albumina e os outros biomarcadores mensurados neste estudo é muito mais exigente que os demais (ETA e $\mathrm{LEP}_{\mathrm{L}}$ ), o que gera maior número de amostras rejeitadas. Isto se deve ao fato de que o LEPc reflete com maior precisão as condições analíticas, sendo, portanto, um critério ideal para estudos investigativos, porém rigoroso demais para o controle de qualidade de exames laboratoriais de rotina.

A bilirrubina total plasmática de cães apresenta valores baixos na presença de concentração de hemoglobina até $1 \mathrm{mg} / \mathrm{L}$. Essa diminuição também foi observada em todas as frações de bilirrubina (total, conjugada e não conjugada). É sabido que a hemoglobina livre interfere na formação da cor por diazônio na mensuração da bilirrubina sérica (Yucel e Dalva, 1992; Koseoglu et al., 2011). Entretanto, o efeito da hemólise na quantificação de bilirrubina é contraditória. Há relato de que concentrações de hemoglobina maiores que $0,8 \mathrm{~g} / \mathrm{L}$ geram resultados de bilirrubina sérica falsamente diminuídos (Sonntag, 1986), porém esse efeito inibidor da diazotização causado pela hemólise não foi observado por outros investigadores (O'neill e Feldman, 1989). Em cães, o presente estudo também não encontrou diferenças significativas na concentração de bilirrubina em amostras com diferentes graus de hemólise, e o erro relativo médio foi muito menor que o ETA e o LEP utilizados para rejeição de resultados.

Tanto em cães (Almeida et al., 2011) como em humanos (Koseoglu et al., 2011; Lippi et al.; 2006), há relatos de que a hemólise causa diminuição da atividade de GGT sérica. No presente estudo, confirmou-se que mesmo um pequeno grau de hemólise não detectável visualmente diminui a atividade de GGT plasmática, causando erro relativo maior que o permitido (LEP).

ASVCP Guidelines Allowable Total Error (Harr et al., 2013) estabelece o ETA como referência para a maioria dos programas de controle de qualidade dos laboratórios clínicos, entretanto não estabelece esses valores para TAC e TOC. Também não foram encontrados, no presente estudo, valores de referência confiáveis para a TAC e a TOC plasmáticas de cão; há apenas valores de pequenos grupos controle de estudos experimentais (Almeida et al., 2013). Há somente dois relatos de que a hemólise em humanos não interfere na quantificação da TAC plasmática (Kampa et al., 2002; Erel, 2004). Constatou-se aqui que pequenas concentrações de hemoglobina $(<0,36 \mathrm{~g} / \mathrm{L})$ causam diminuição da TAC plasmática de cães e erro relativo maior 
que o LEP. O eritrócito é uma célula transportadora de oxigênio e detém um rico sistema para evitar lesão oxidativa (Tedesco et al., 2000), portanto inicialmente houve a hipótese de que, após a hemólise, a liberação dos antioxidantes intraeritrocitários seria potencialmente responsável por um aumento da TAC plasmática. Desse modo, é razoável supor que, neste caso, a fonte do erro não esteja relacionada ao acréscimo de antioxidante e do pigmento hemoglobínico, mas a alguma inibição da reação entre os antioxidantes e o ABTS utilizado no método de Erel (2004), provavelmente uma alteração de $\mathrm{pH}$. Pode-se ainda especular que o efeito oxidativo causado pela hemólise tenha sido predominante, uma vez que mesmo pequeno grau hemolítico causou aumento significativo da TOC plasmática, gerando erros ainda maiores em relação ao LEP.

O emprego dos resultados do presente estudo deve ser limitado aos casos de hemólise mecânica in vitro e não a outros tipos, como as doenças hemolíticas. Portanto, os erros calculados podem ser considerados para a maioria dos casos de hemólise observada na rotina laboratorial, visto que esses casos reproduzem a lise mecânica, principal causa de rejeição de amostra (Almeida et al., 2011).

Neste estudo foi confirmado que mesmo um pequeno grau de hemólise gera algum erro analítico não aceitável (ETA e/ou LEP) em todos os biomarcadores plasmáticos de estresse oxidativo mensurados, exceto a bilirrubina.

\section{CONCLUSÃO}

A hemólise é um importante fator que limita a avaliação do estresse oxidativo sistêmico mensurado no plasma, podendo causar erros que potencialmente comprometam o diagnóstico clínico.

\section{REFERÊNCIAS}

ALMEIDA, B.F.; NARCISO, L.G.; MELO, L.M. et al. Leishmaniasis causes oxidative stress and alteration of oxidative metabolism and viability of neutrophils in dogs. Vet. J., v.198, p.599-605, 2013.
ALMEIDA, B.F.; ZUCATTO, A.S.; VIEIRA, R.F.C. et al. Efeito da hemólise sobre o perfil bioquímico sérico canino, bovino e equino. Med. Vet., v.5, p.12-17, 2011.

BOSCO, A.M.; PEREIRA, P.P.; ALMEIDA, B.F.M. et al. Free p-Cresol alters neutrophil function in dogs. Artif. Organs, v.40, p.480-488, 2016.

CARRARO, P.; SERVIDIO, G.; PLEBANI, M. Hemolyzed specimens: a reason for rejection or a clinical challenge? Clin. Chem., v.46, p.306-307, 2000.

CLINICAL Laboratory Improvement Amendments of 1988. Stat 42 USC 201. H.R. 5471, 1988. (Public Law 100-578).

CIMEN, BURAK, M.Y. Free radical metabolism in human erythrocytes. Clin. Chim. Acta, v.390, p.1-11, 2008.

DURAK, I.; KAÇMAZ, M.; ÇIMEN, M.Y.B. et al. Blood oxidant/antioxidant status of atherosclerotic patients. Int. J. Cardiol., v.77, p.293-297, 2001.

EREL, O. A novel automated direct measurement method for total antioxidant capacity using a new generations, more stable ABTS radical cation. Clin. Biochem., v.37, p.277-285, 2004.

EREL, O. A new automated colorimetric method for measuring total oxidant status. Clin. Biochem., v.38, p.1103-1111, 2005.

HARR, K.E.; FLATLAND, B.; NABITY, M. et al. ASVCP guidelines: allowable total error guidelines for biochemistry. Vet. Clin. Path., v.42, p.424-436, 2013.

JACOBS, R.M.; LUMSDEN, J.H.; GRIFT, E. Effect of bilirrubinemia, hemolysis, and lipemia on clinical chemistry analytes in bovine, canine, equine and feline sera. Can. Vet. J., v.33, p.605608, 1992.

KAMPA, M.; NISTIKAKI, A.; TSAOUSIS, V. et al. A new automated method for the determination of the Total Antioxidant Capacity (TAC) of human plasma, based on the crocin bleaching assay. BMC Clin. Pathol., v.2, p.1-16, 2002.

KANEKO, J.J.; HARVEY, J.W.; BRUSS, M.L. (Eds.) Clinical biochemistry of domestic animals. [Washington]: Academic press, 2008. 928p. 
KOSEOGLU, M.; HUR, A.; ATAY, A. et al. Effects of hemolysis interferences on routine biochemistry parameters. Biochem. Med., v.21, p.79-85, 2011.

LEARD, B.L.; ALSAKER, R.D.; PORTER, W.P. et al. The effect of haemolysis on certain canine serum chemistry parameters. Lab. Anim., v.24, p.32-35, 1990.

LIPPI, G.; SALVAGNO, G.L.; MONTAGNANA, M. et al. Influence of hemolysis on routine clinical chemistry testing. Clin. Chem. Lab. Med., v.44, p.311-316, 2006.

LIPPI, G.; BLANCKEAERT, N.; BONINI, P. et al. Haemolysis: an overview of the leading cause of unsuitable specimens in clinical laboratories. Clin. Chem. Lab. Med, v.46, p.764-772, 2008.

LIPPI, M.; PLEBANI, S.D.; CERVELLIN, G. Hemolyzed specimens: a major chalenge for emergency departaments and clinical laboratories. Crit. Rev. Clin. Lab. Sci., v.48, p.143-153, 2011.

MENDES, M.E.; ROMANO, P. Validação de sistema analítico. Gestão da fase analítica do laboratório: como assegurar a qualidade na prática. ControlLab, p.119-143, 2010.

OLIVEIRA, C.A.; MENDES, M.A.; Gestão da fase analítica do laboratório como assegurar a qualidade na prática. ControlLab, v.1, p.1-146, 2010 .

O'NEILL, S.L.; FELDMAN, B.F. Hemolysis as a factor in clinical chemistry and hematology of the dog. Vet. Clin. Path., v.18, p.58-68, 1989.

PEAKE, J.; SUZUKI, K. Neutrophil activation, antioxidant supplements and exercise-induced oxidative stress. Exerc. Immunol. Rev., v.10, p.129-141, 2004.
SIMUNDIC, A.M.; NIKOLAC, N.; IVANKOVIC, V. et al. Comparison of visual vs. automated detection of lipemic, icteric and hemolyzed specimens: can we rely on a human eye? Clin. Chem. Lab. Med., v.47, p.1361-1365, 2009.

SONNTAG, O. Haemolysis äs an interference factor in clinical chemistry. Clin. Chem., v.24, p.127-139, 1986.

TAVAZZI, B.; PIERRO; D.D., AMORINI, A.M. et al. Energy metabolism and lipid peroxidation of human erythrocytes as a function of increased oxidative stress. Eur. J. Biochem., v.267, p.684689,2000

TEDESCO, I.; RUSSO, M.; RUSSO, P. et al. Antioxidant effect of red wine polyphenols on red blood cells. J. Nutr. Biochem., v.11, p.114$119,2000$.

TONKS, D.B. A study of accuracyand precision of clinical chemistry determinations in 170 Canadian laboratories. Clin. Chem., v.9, p.217233, 1963.

WESTGARD, J.O.; DARCY, T. The truth about quality: medical usefulness and analytical reability of laboratory tests. Clin. Chim. Acta, v.346, p.3-11, 2004.

YÜCEL, D.; DALVA, K. Effect of in vitro hemolysis on 25 common biochemical testes. Clin. Chem., v.38, p.575-577, 1992.

ZAMZAMI, N; MARCCHETTI, P.; CASTEDO, M. et al. G. Inhibitors of permeability transition interfere with the disruption of the mitochondrial transmembrane potential during apoptosis. FEBS Lett., v.384, p.53-57, 1996. 